\section{The Frequency of Antipsychotic Prescribing in Older People Mental Health Services: A Southern Health OPMH CRIS Audit}

\section{Abstract}

Background: Antipsychotic prescription in older people with mental health problems can have severe adverse effects such as an increased risk of falls and show higher mortality rates. Moreover, this risk is elevated for the elderly with dementia.

Aims: to provide an estimate of the frequency of antipsychotic use for mental health problems in older people mental health (OPMH) services and collect evidence of adherence to NICE guidelines.

Methods: A retrospective clinical audit of typical and atypical antipsychotic medication use in OPMH services. A clinical Research Interactive Search (CRIS: 2007) was conducted from $1^{\text {st }}$ January 2016 until $31^{\text {st }}$ December 2017 . This focused on patients referred to OPMH services that were being prescribed Flupentixol, Haloperidol, Prochloperazine, Amisulpride, Olanzapine, Quetiapine or Risperidone.

Results: There were $1,642(22.75 \%)$ patients prescribed one or more of the seven selected antipsychotics within the OPMH services. Of these patients without comorbid psychotic illness 1390 (84.6\%) were prescribed an antipsychotic; the most common indications for such medication were agitation, psychotic symptoms, distress or aggression. This is $22.75 \%$ of the total number of people currently under OPMH services and is not limited to those of a specific disorder. The most commonly prescribed were atypicals and specifically Risperidone. The most common diagnosis prescribed antipsychotic medication was dementia $40 \%$.

Conclusions: These prescribing behaviours reveal good practice, in line with current NICE guidelines of what would be expected for the OPMH services. However, a further investigation into the rationale for the prescribing behaviour evident and the quality of alternative pharmacological and non-pharmacological approaches available to this population is essential.

Keywords: Audit; Antipsychotics; Older people mental health; Dementia; Psychosis; Schizophrenia; Flupentixol; Haloperidol; Prochloperazine; Amisulpride; Olanzapine; Quetiapine; Risperidone

\section{Peter Phiri, ${ }^{1,2 *}$, Hannah Carr ${ }^{1}$ and Shanaya Rathod ${ }^{1}$
1 Southern Health NHS Foundation Trust, UK Health Environment, University of Southampton, UK \\ 2 School of Health Sciences, Faculty of}

*Corresponding author: Dr. Peter Phiri, PhD

” peter.phiri@nhs.net

R\&D Manager, Southern Health NHS Foundation Trust, Research \& Development, Clinical Trials Facility, Tom Rudd Unit, Moorgreen Hospital, Botley Road, West End Southampton, UK.

Tel: 02380475112

Citation: Phiri P, Carr H, Rathod S (2019) The Frequency of Antipsychotic Prescribing in Older People Mental Health Services: A Southern Health OPMH CRIS Audit. Acta Psychopathol Vol.5 No.1:2

Received: December 03, 2018; Accepted: March 18, 2019; Published: March 25, 2019

\section{Introduction}

Antipsychotic medications are used to treat the psychotic symptoms of a variety of mental health conditions such as Schizophrenia, Schizoaffective and psychosis. They can also be used to treat the agitation and psychotic experiences often presented in dementia. There are two forms of antipsychotic medication; first generation (typical) and second generation (atypical). Typical antipsychotics were introduced first but atypical antipsychotics were later introduced and are now more commonly prescribed due to their favourable side-effect and safer metabolic profiles [1-4].

One population, which may be at a higher risk of adverse events 
and side-effects from antipsychotics are the elderly. Age related changes in pharmacokinetics and pharmacodynamics results in an increased sensitivity to drugs and their side effects [5]. Therefore, it is important to be mindful of prescribing behaviours to the elderly especially when prescribing antipsychotics (both typical and atypical), which are shown to produce some severe adverse events and side-effects. For example, Landi [1] has shown an increased rate of falls by nearly $47 \%$ in the elderly being prescribed antipsychotics. It has also been shown that the risk of ischemic stroke is just as likely in those taking typical as atypical antipsychotics [6], the risk of falls [7] and similar results were also seen when comparing mortality rates and risk of death [8] However, the risk of death associated with typical antipsychotics is comparable to and possibly greater than the risk of death associated with atypical antipsychotic medications [9]. Likewise in dementia the association between antipsychotic use and increased morbidity and mortality is known Barnes et al.

Although atypical antipsychotic drugs are more commonly used they still present side-effects and adverse events. For example, they have been associated with higher hospitalisation rates with acute kidney injury [8], an increased 90 day risk of non-vertebral osteoporatic fracture, hip fracture and various other fractures and falls [10] and also with an associated marginal increased risk of death compared to those on a placebo [11]. A clinical trial has also failed to show either safety or effectiveness of four specific antipsychotics (aripiprazole, olanzapine, quetiapine, and risperidone) in both middle aged and older patients with a variety of psychotic disorders [12]. When comparisons have been made between specific antipsychotic medications, it appears that some may also produce more adverse events than others. For example, Risperidone has not been reported to cause anticholinergic sideeffects in the elderly but clozapine has been implicated [13].

It thus appears that both forms of antipsychotics produce sideeffects or adverse events that need to be taken into consideration when prescribing to the elderly. A more recent systematic review, however, has shown that the use of antipsychotics was not related to an increased risk of falls in ten studies. There was the exception of a single study with a very old ( $>85$ years old) home-dwelling population, which showed an elevated risk of falls [14]. Therefore, there may be variables such as age that affect the risk of adverse events in elderly populations taking antipsychotic medications.

Nonetheless, the research appears to suggest that antipsychotic medication in elderly populations may have possible side-effects that should be taken into consideration. In a particular elderly population (those with dementia) it has been argued in a report commissioned by the minister of health that these medications should only be prescribed when absolutely necessary $[2,15]$. This audit thus aims to highlight the number of typical and atypical antipsychotics used in the OPMH services in light of National Institute for Health and Clinical Excellence (NICE) guidelines and good practice standards.

\section{Methodology}

The present data was extracted from anonymised, electronic heath records of those using the OPMH services in Southern Health NHS Foundation Trust between $1^{\text {st }}$ January 2016 and $31^{\text {st }}$ December 2017. The data was extracted using Southern Health's Clinical Records Interactive Search (UK-CRIS) in January 2018. UK-CRIS is a computer system that unlocks and transforms clinical data held in Trust electronic system to provide a rich and pseudonymised ${ }^{1}$ resource enabling clinicians and researchers (with a valid research passport, honorary or substantive contract with the Trust) to interrogate de-identified patient data directly through a secure data repository investigate hypotheses and identify patient cohorts (ref) This searched through both structured and free text fields. The structured fields included were: date of birth, ethnicity, gender, referral team, created date of progress note, diagnosis and created date of diagnosis and medications prescribed and dosage. The free text field analysed was the progress note text.

\section{Study sample}

The study included an initial sample of 3,064 adults who were under the care of the OPMH services within Southern Health from $1^{\text {st }}$ January 2016 until $31^{\text {st }}$ December 2017 and who had a mention of an antipsychotic drug within their progress notes. From this, the sample was reduced, removing those who were not currently taking an antipsychotic (having been discontinued or only mentioned possible future use etc.). This was done manually by going through each individual's progress notes. The sample then consisted of a total of 1642 service users identified as being prescribed one or more of the antipsychotic medication between the specified time periods. Some of these were taking multiple antipsychotics in this period and for the purpose of this audit, only the most recent antipsychotics prescribed were included. The demographic and clinical characteristics of the sample are shown in Table 1.

\section{Measurements}

\section{Outcome: antipsychotic use}

Only the top seven antipsychotics prescribed are included in this audit. Those not included were prescribing to less than 25 patients.

Once all cases of prescribing were identified for both typical and atypical antipsychotics these were then cross-referenced to a) identify when one medication had been replaced with another and b) to identify when service users were being prescribed multiple antipsychotics.

Data gathered regarding antipsychotic use included the type of antipsychotic, the dose and frequency.

\section{Statistical analysis}

Data were analysed using Windows package and SPSS Analysis involved descriptive data, frequency and descriptive statistics were utilised to determine the frequencies of antipsychotic use.

${ }^{1}$ UK-CRIS data is pseudonymised with a randomly generated pseudonym that is not linked to any data within the record so the pseudonym is impervious to attack. 
Table 1: A table showing the demographic information of the $\mathrm{OPMH}$ service users involved in this audit.

\begin{tabular}{|c|c|c|c|}
\hline & \multirow[b]{2}{*}{$\begin{array}{l}\text { Total sample } \\
(n=1642)^{*}\end{array}$} & \multicolumn{2}{|c|}{$\begin{array}{l}\text { Antipsychotic Prescribed }(n= \\
1642)\end{array}$} \\
\hline & & $\begin{array}{c}\text { First } \\
\text { Generation } \\
(n=244)\end{array}$ & $\begin{array}{c}\text { Second } \\
\text { Generation } \\
(n=1580)\end{array}$ \\
\hline \multicolumn{4}{|l|}{ Gender } \\
\hline Male & 612 & 81 & 595 \\
\hline Female & 1,029 & 143 & 983 \\
\hline Not Specified & 1 & - & 1 \\
\hline \multicolumn{4}{|l|}{ Ethnicity } \\
\hline White-British & 1154 & 175 & 1037 \\
\hline $\begin{array}{l}\text { White - any other } \\
\text { background }\end{array}$ & 34 & 7 & 30 \\
\hline $\begin{array}{l}\text { Asian or Asian } \\
\text { British }\end{array}$ & 11 & 4 & 7 \\
\hline $\begin{array}{c}\text { Black or Black } \\
\text { British }\end{array}$ & 2 & 2 & - \\
\hline Not Known & 437 & 35 & 405 \\
\hline Other & 4 & 1 & 3 \\
\hline \multicolumn{4}{|l|}{ Age } \\
\hline $\begin{array}{c}\text { Mean (SD) } \\
\text { Median } \\
\text { Interquartile range }\end{array}$ & $\begin{array}{c}78.97(8.78) \\
79 \\
12\end{array}$ & $\begin{array}{c}77.13(8.91) \\
77 \\
12\end{array}$ & $\begin{array}{c}79.08(8.69) \\
79 \\
12\end{array}$ \\
\hline $\begin{array}{l}\text { Minimum - } \\
\text { Maximum }\end{array}$ & $43-99$ & $53-99$ & $43-99$ \\
\hline \multicolumn{4}{|l|}{ Age, $n(\%)$} \\
\hline $\begin{array}{l}65 \text { years or } \\
\text { younger }\end{array}$ & 75 & 12 & 63 \\
\hline 66-74 & 447 & 77 & 400 \\
\hline 75-84 & 647 & 88 & 580 \\
\hline 85 and older & 456 & 44 & 417 \\
\hline Not Stated & 17 & - & 17 \\
\hline \multicolumn{4}{|l|}{ Diagnosis } \\
\hline Dementias & 651 & 61 & 601 \\
\hline Psychotic Disorders & 142 & 58 & 114 \\
\hline Mood Disorders & 295 & 32 & 277 \\
\hline Dementia Mixed & 118 & 21 & 102 \\
\hline Mixed Other & 175 & 19 & 159 \\
\hline Other & 111 & 17 & 96 \\
\hline Not Stated & 150 & 17 & 134 \\
\hline
\end{tabular}

*Total sample refers to the individuals recruited into this study - some participants were taking both first and second generation antipsychotics so this figure can be less than that of each generation of antipsychotic.

\section{Results}

The top seven prescribed antipsychotics were identified. The number of service users being prescribed each of these is shown in Figure 1.

In total, 1,642 patients were identified as being prescribed one of the seven antipsychotics during the 24 month observational period. This is $22.75 \%$ of all open referrals to OPMH at this time. Of the 1,642 patients, 65 were prescribed at least one typical and one atypical simultaneously. A further 43 were prescribed two or more antipsychotics simultaneously.

\section{Dosage}

The mean daily dosage ranged from $0.95 \mathrm{mg}-305.4 \mathrm{mg}$. The antipsychotic prescribed with the highest mean dose was Amisulpride (317.14 mg). The antipsychotic prescribed with the lowest dose on average was Risperidone $(0.95 \mathrm{mg})$. The mean dose for each individual antipsychotic can be seen in Table 2.

\section{Discussion}

Overall, the current audit shows that $22.75 \%$ of service users within the OPMH service of Southern Health NHS Foundation Trust were being prescribed antipsychotics between January 2016 and December 2017. Of the different antipsychotics, it appears that atypical were more often prescribed and of them, Risperidone was prescribed the most. Service users were most commonly prescribed antipsychotic with a dementia diagnosis. Clinically, these results would fit with the rationale to prescribe atypical antipsychotics more so than typical antipsychotics to this population. More specifically, the greater number of Risperidone prescriptions to this population and more so to those diagnosed with dementia is in line with the licensing of Risperidone as the only antipsychotic to be prescribed for dementia.

When comparing the doses prescribed within Southern Health's OPMH service to that recommended by BNF, it appears that all have a mean dosing within these recommendation, when looking at their ranges, however, most of the antipsychotics audited appear to have upper dosing ranges much greater than that recommended. For example, Haloperidol Decanoate has a recommendation of $25-75 \mathrm{mg}$ every 4 weeks but the highest dose recorded on patient's progress notes is $200 \mathrm{mg}$. The BNF guidelines do acknowledge higher doses and state that "doses above 75 mg every 4 weeks should only be considered in patients who have tolerated higher doses and after reassessment of the individual benefit-risk, if supplementation with oral haloperidol is required, the combined total dose of haloperidol from both formulations must not exceed the corresponding maximum oral haloperidol dose of $5 \mathrm{mg}$ per day, or the previously administered oral haloperidol dose in patients who have received long-term treatment with oral haloperidol". Although in some cases higher doses are acknowledged and recommended by the BNF and NICE the rationale behind these prescriptions in the current population should be further analysed to investigate if this behaviour fits with the recommendation of only being considered when patients have a higher tolerance and have been reassessed.

From the data, it must be highlighted that typical antipsychotics, though much less frequent, are still being prescribed for a range of disorders. One possibility is that this population may have been prescribed a typical antipsychotic many years previously and have remained on them for continuity and efficacy of the drug. They may also be combining their previous typical with a new atypical for other symptoms that are being presented such as those related to a dementia diagnosis. For example, service users may have been prescribed a Flupentixol depot over a long period of time and are now combining this with Risperidone for new cognitive decline symptoms. This is, however, only one possibility. Although this is an audit, a future study may be useful 


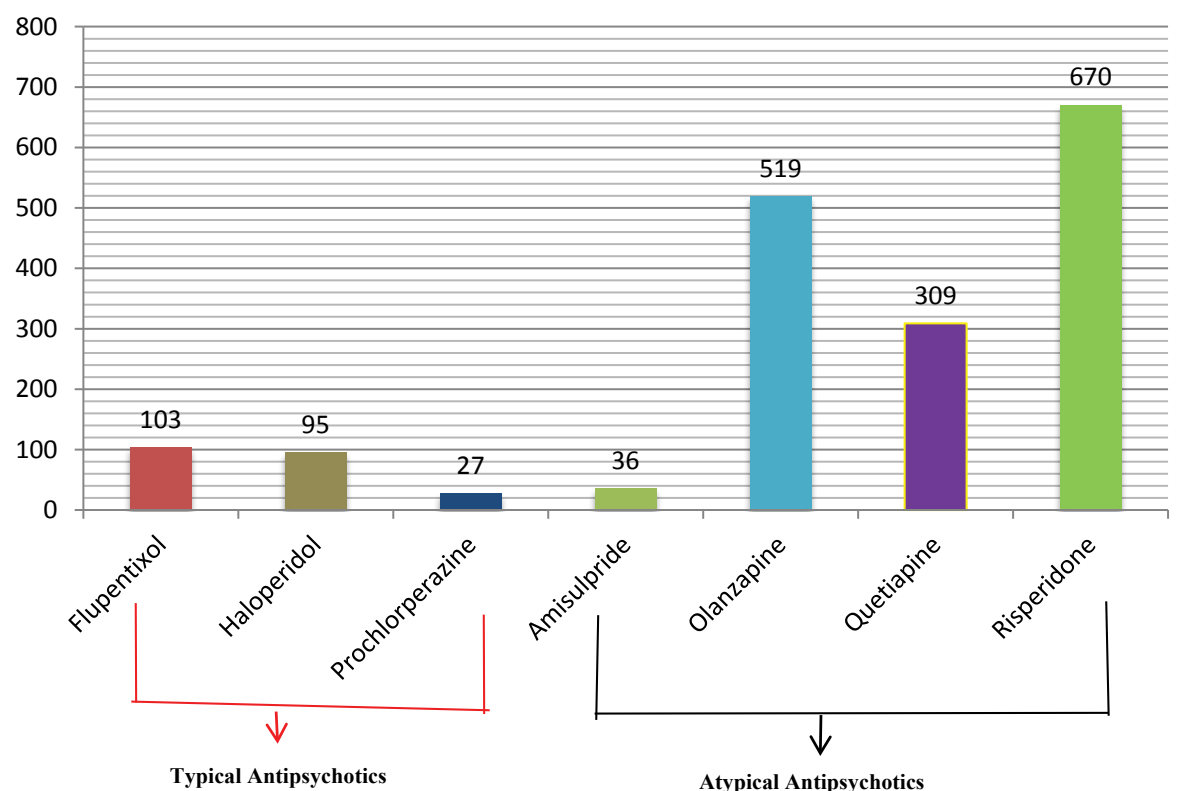

Figure 1 A figure showing the number of OPMH service users being prescribed each antipsychotic medication.

Table 2: A table showing the dosing range, mean (SD) of each of the seven prescribed antipsychotics and the BNF recommendations for each.

\begin{tabular}{|c|c|c|c|}
\hline Antipsychotic & Range (mg) & $\begin{array}{l}\text { Mean Daily Dose in mg } \\
\text { (SD) }\end{array}$ & BNF/NICE Guidelines for Elderly \\
\hline Flupentixol & $.5-10.0$ & $2.21(2.55)$ & $0.75-4.5 \mathrm{mg}$ twice daily, adjusted according to response \\
\hline Flupentixol Decanoate & $1.0-225.0$ & $34.74(43.39)$ & $\begin{array}{l}\text { Dose is initially quarter to half adult dose (maximum 100- } \\
\qquad 200 \mathrm{mg} \text { per week) }\end{array}$ \\
\hline Haloperidol & $.5-7.0$ & $1.64(1.51)$ & $5 \mathrm{mg}$ daily \\
\hline Haloperidol Decanoate & $5.0-200.0$ & $70.25(70.51)$ & $25-75 \mathrm{mg}$ every 4 weeks \\
\hline Prochlorperazine & $3.0-500.0$ & $33.32(104.34)$ & $75-100 \mathrm{mg}$ daily orally*, $12.5-25 \mathrm{mg} 2-3$ times a day IM* \\
\hline Amisulpride & $25.0-1200.0$ & $317.14(359.62)$ & maximum $1.2 \mathrm{~g}$ per day* \\
\hline Olanzapine & $.5-50.0$ & $6.55(4.79)$ & maximum daily combined oral and parenteral dose $20 \mathrm{mg}$ \\
\hline Quetiapine & $2.50-800.0$ & $120.32(141.31)$ & $\begin{array}{l}\text { Initially } 50 \mathrm{mg} \text { once daily, adjusted according to response. } \\
\text { adjusted in steps of } 50 \mathrm{mg} \text { daily }\end{array}$ \\
\hline Risperidone & $.25-15.0$ & $.95(1.08)$ & $1-2 \mathrm{mg}$ twice daily \\
\hline Risperidone Depot & $25.0-25.0$ & $25.0(0)$ & max. per dose 50 mg every 2 weeks* \\
\hline
\end{tabular}

*Where no recommendation for the elderly is available the adult recommendation is provided.

in identifying the rationale behind the prescribing behaviour to ensure that this prescribing behaviour is warranted. It may also be beneficial to identify if the prescribing of these typical antipsychotics is associated with any of the side-effects or adverse events that have been previously reported in the literature.

Furthermore, other atypical other than Risperidone are also being prescribed for those with a dementia diagnosis. As Risperidone is the only licensed antipsychotic for this population, it must be identified why this is occurring. It may be the case that Risperidone has previously been prescribed but has not been effective and therefore an alternative has been prescribed. As stated previously, there were many cases of multiple antipsychotics being prescribed in the two years of data explored but only the most recent was reported. It may be useful to identify the number of changes in antipsychotic medication in this population and if, for those with dementia, Risperidone is the first option and others are later alternatives.

Another interesting point to be made is that the majority of the population in this audit are of White-British nationality. This is to be expected for the geographical location of the Trust investigated. It would be interesting to now compare these results with a Trust in a more diverse area such as London. Previous literature has suggested that there are significant differences in antipsychotic prescribing between White British and those of a Black and Minority ethnic groups $[16,17]$. This could, therefore, provide a useful comparator to ensure that other Trusts with various populations are still adhering to prescribing regulations regardless of factors such as ethnicity.

The NHS has a busy workforce and clinical caseload resulting in insufficient participation in clinical audits such as the POMH-UK; 
this is often despite concentrated efforts by organisations to raise awareness and engaging clinical services participation. The collection and collation of clinical health record data for audit purposes can be burdensome for clinicians and does impact on 'time to care'. There are varied approaches to audits within the organisation, some of which use electronic health records (e.g. OpenRiO system) and others that rely solely on manual review of medical case records and treatment charts that are captured on data capture sheets, or indeed conducting word searches on clinical systems on individual case notes, this is often time consuming and an onerous task. In contrast, CRIS provides an invaluable tool for service evaluation, clinical audits and research. It enables researchers to interrogate a large number of de-identified patient case record data directly and efficiently through a secure data repository.

It extracts relevant clinical records based on the clinician's query. The clinician can specify the fields to display, these may be the same as or different from those they searched against. On running a query, CRIS displays how many patients match the chosen criteria and returns results in a spreadsheet view. The clinician can then export results as CSV files for further analysis. Queries can be saved for future use, therefore saving clinician's time. Furthermore, all searches in CRIS have an audit trail for monitoring compliance with the security protocols. CRIS increases efficiency in that the platform provides faster delivery of results from audit or research initiatives and leads to earlier improvements in patient care. CRIS has implications for reducing staff time spent on conducting audits therefore reducing costs associated with these activities. Additionally, by using CRIS the output is faster and can direct research that meets the local needs of the population. Finally, CRIS raises frontline awareness of the value of collecting high quality clinical information for quality improvement and translational research benefit. However, a potential limitation of CRIS data in our organisation is the exclusion of attachments which are currently not accessible to the CRIS system.

\section{Strengths and limitations of the audit}

There is much strength to this audit including the access to a large amount of rich data. From this audit we were able to identify in progress notes when antipsychotic medication was ended, changed or combined. This has allowed the data presented to be as accurate as possible. It also presents the data to everyone who has not opted out of CRIS. This is a large proportion of those within $\mathrm{OPMH}$ and therefore can provide generalizable data for the Trust's antipsychotic prescribing to the elderly.

One must be cautious when interpreting the results, however. The data was primarily extracted from free text data (progress notes) with over 300,000 progress notes available. This is strength, as it provides a large amount of rich data but this had to be manually coded as natural language processing (NLP) software was not available to do this. With manual coding there is the risk of human error such as missing information in a progress note that may be important to the data. This audit may thus benefit from using NLP in the future to improve both the validity and also the time taken to analyse the data.
Furthermore, essential data is often placed in many different fields in OpenRIO and all fields are not always populated. This therefore makes it harder to identify the required data and therefore data was extracted from multiple fields. This can often be contradictive if one field is often updated and the other is not. For example, the diagnosis field in OpenRIO is often only updated after an inpatient admission this may not be updated therefore as regularly as the diagnosis within the progress note text. With progress note text, however, it can often be inputted by a nonmedical professional who may input an incorrect diagnosis. Therefore, the accuracy of the data itself can sometimes be an issue and this must be considered in line with the data presented here.

\section{Implications for quality improvement}

Nonetheless, these findings may have implications in the monitoring of antipsychotic prescribing behaviour within the OPMH services in the Trust. In light of the literature suggesting that antipsychotics may have negative impacts on this population, it is useful to identify if the prescribing behaviour is occurring with sensitivity to the population's needs. That is, ensuring that they are being prescribed only when necessary to prevent the risk of falls, fractures, other adverse events and ultimately death. Regular audits may thus be useful to ensure that best practise is occurring and that patient's safety is continuously monitored and kept as a top priority.

\section{Implications for future practice}

This audit allows for the possibility of future qualitative research to be conducted. It must be highlighted why certain prescribing behaviours are occurring (i.e. is there a clear rationale for prescribing Flupentixol rather than Risperidone?). If there is no clear scientific rationale for this prescribing behaviour then education may need to be improved amongst OPMH clinicians to ensure that their prescribing is adhering to BNF guidelines, has a clear rationale and is preventing side-effects and adverse reactions in patients as much as possible. This clinical audit will allow OPMH services to reflect on their prescribing behaviours of antipsychotic prescribing in older people with mental health problems and dementias in particular to consider alternative approaches given that practice behaviour was relatively good and within the NICE recommendations.

\section{Funding}

None

\section{Acknowledgements}

The authors would like to thank Dr. Sarah Constantine, Consultant Psychiatrist, Associate Medical Director for requesting the Audit and supporting CRIS in OPMH. "This study was supported by the UK Clinical Record Interactive Search (UK-CRIS) system funded and developed by the National Institute for Health Research (NIHR), the Medical Research Council and the University of Oxford, using the system within Southern Health NHS Foundation Trust." 


\section{References}

1 Landi F, Onder G, Cesari M, Barillaro C, Russo A, et al. (2005) Psychotropic medications and risk for falls among communitydwelling frail older people: an observational study. J Gerontol A Biol Sci Med Sci 60: 622-626.

2 Banerjee S (2009) The use of antipsychotic medication for people with dementia: time for action. A report for the Minister of State for Care Services. United Kingdom Department of Health.

3 Kale HC, Kim HM, Zivin K, Valenstein M, Seyfried LS, et al. (2012) Risk of mortalilty among individual antipsychotics in patients with dementia. Am J Psychiatry 169: 71-79.

4 Solmi M, Murru A, Pacchiarotti I, Undurraga J, Veronese $\mathrm{N}$, et al. (2017) Safety, tolerability, and risks associated with first-and second-generation antipsychotics: a state-of-the-art clinical review. Ther Clin Risk Manag 13: 757-777.

5 Mangoni AA, Jackson SH (2004) Age-related changes in pharmacokinetics and pharmacodynamics: basic principles and practical applications. Br J Clin Pharmacol 57: 6-14.

6 Rochon PA, Stuke TA, Sykora K, Gill S, Garfinkel S, et al. (2005) Atypical antipsychotics and parkinsonism. Arch Intern Med 165: 1882-1888.

7 Bakken MS, Schjøtt J, Engeland A, Engesæter LB, Ruths S (2016) Antipsychotic drugs and risk of hip fracture in people aged 60 and older in Norway. J Am Geriatr Soc 64: 1203-1209.

8 Hwang YJ, Dixon SN, Reiss JP, Wald R, Parikh CR, et al. (2014) Atypical antipsychotic drugs and the risk for acute kidney injury and other adverse outcomes in older adults: a population-based cohort study. Ann Intern Med 161: 242-248.
9 Schneeweiss S, Setoguchi S, Brookhart A, Dormuth C, Wang PS (2007) Risk of death associated with the use of conventional versus atypical antipsychotic drugs among elderly patients. CMAJ 176: 627-632.

10 Fraser LA, Liu K, Naylor KL, Hwang YJ, Dixon SN, et al. (2015) Falls and fractures with atypical antipsychotic medication use: a populationbased cohort study. JAMA Intern Med 175: 450-452.

11 Schneider LS, Dagerman KS, Insel P (2005) Risk of death with atypical antipsychotic drug treatment for dementia: meta-analysis of randomized placebo-controlled trials. JAMA 294: 1934-1943.

12 Jeste DV, Maglione JE (2013) Atypical antipsychotics for older adults: are they safe and effective as we once thought? J Comp Eff Res 2: 355-358.

13 Mintzer J, Burns A (2000) Anticholinergic side-effects of drugs in elderly people. J R Soc Med 93: 457-462.

14 Park Y, Franklin JM, Schneeweiss S, Levin R, Crystal S, et al. (2015) Antipsychotics and mortality: adjusting for mortality risk scores to address confounding by terminal illness. J Am Geriatr Soc 63: 516523.

15 NICE (2006) Dementia. Guideline on supporting people with dementia and their carers in health and social care (Clinical guidance CG42) (http://www.nice.org.uk/nicemedia/live10998/30318/30318.pdf).

16 Bécares L, Dewey ME, Das-Munshi J (2018) Ethnic density effects for adult mental health: systematic review and meta-analysis of international studies. Psychol Med 48: 2054-2072.

17 Schofield P, Thygesen M, Das-Munshi J, Becares L, Cantor-Graae E, et al. (2018) Neighbourhood ethnic density and psychosis-ls there a difference according to generation? Schizophr Res 195: 501-505. 